\title{
THE RECTIFYING DEVELOPABLE AND THE SPHERICAL DARBOUX IMAGE OF A SPACE CURVE
}

\author{
SHYUICHI IZUMIYA \\ Department of Mathematics, Hokkaido University \\ Sapporo 060-0810, Japan \\ E-mail: izumiya@math.sci.hokudai.ac.jp \\ HARUYO KATSUMI and TAKAKO YAMASAKI \\ Department of Mathematics, Ochanomizu University \\ Bunkyou-ku Otsuka Tokyo 112-8610, Japan
}

Dedicated to the memory of Professor Yosuke Ogawa

\begin{abstract}
In this paper we study singularities of certain surfaces and curves associated with the family of rectifying planes along space curves. We establish the relationships between singularities of these subjects and geometric invariants of curves which are deeply related to the order of contact with helices.

1. Introduction. There are several articles concerning singularities of the tangent developable (i.e., the envelope of osculating planes) and the focal developable (i.e., the envelope of normal planes) of a space curve ([3-12]). In these papers the relationships between singularities of these surfaces and classical geometric invariants of space curves have been studied. The notion of the distance-squared functions on space curves is useful for the study of singularities of focal developable $[7,10,11]$. For tangent developable, there are other techniques to study singularities $[3-6,8,9]$. The classical invariants of extrinsic differential geometry can be interpreted as "singularities" of these developable; however, the authors cannot find any article concerning singularities of the rectifying developable (i.e., the envelope of rectifying planes) of a space curve. The rectifying developable is an important surface in the following sense: the space curve $\gamma$ is always a geodesic of the rectifying developable of itself (cf. [2, p. 308]).
\end{abstract}

1991 Mathematics Subject Classification: Primary 58C27; Secondary 53A04.

The paper is in final form and no version of it will be published elsewhere. 
In this paper we introduce the notion of volumelike distance functions (or, binormal directed distance functions) on space curves. This function is quite useful for the study of generic singularities of rectifying developable of space curves. We also introduce the notion of volumelike height functions (or, tangential height functions) on space curves, which induce the notion of rectifying Gaussian surfaces and spherical Darboux images and these singularities are deeply related to the geometry of spherical tangential images of curves.

As a consequence, we apply ordinary techniques of singularity theory for these functions and describe the relationships between the singularities of the above three subjects and differential geometric invariants of space curves.

The main results in this paper are Theorems 2.1 and 2.2. We describe the geometric meaning of Theorem 2.2 in Section 4. The proof of Theorem 2.2 is given in Section 5. In Section 6 we consider generic properties of space curves. Since the calculations for the proof of Theorem 2.1 are terribly long and tedious, we only give a sketch of the proof in this paper. The basic techniques we used in this paper depend heavily on those in the book of Bruce and Giblin [1], so the authors are grateful to both of them. The authors also wish to thank Professors K. Tsukada and T. Fukui for valuable suggestions.

All curves and maps considered here are of class $C^{\infty}$ unless otherwise stated.

2. Basic notions and the main results. We now review some basic concepts on classical differential geometry of space curves in Euclidean space $\mathbb{R}^{3}$. For any two vectors $\boldsymbol{x}=\left(x_{1}, x_{2}, x_{3}\right)$ and $\boldsymbol{y}=\left(y_{1}, y_{2}, y_{3}\right)$, we denote by $\langle\boldsymbol{x}, \boldsymbol{y}\rangle$ the standard inner product. Let $\gamma: I \rightarrow \mathbb{R}^{3}$ be a curve with $\dot{\gamma}(t) \neq 0$, where $\dot{\gamma}(t)=d \gamma / d t(t)$. We also denote the norm of $\boldsymbol{x}$ by $\|\boldsymbol{x}\|$. The arc-length of a curve $\gamma$, measured from $\gamma\left(t_{0}\right), t_{0} \in I$, is

$$
s(t)=\int_{t_{0}}^{t}\|\dot{\gamma}(t)\| d t .
$$

Then a parameter $s$ is determined such that $\left\|\gamma^{\prime}(s)\right\|=1$, where $\gamma^{\prime}(s)=d \gamma / d s(s)$. So we say that a curve $\gamma$ is parametrized by the arc-length if it satisfies $\left\|\gamma^{\prime}(s)\right\|=1$. We define $\boldsymbol{t}(s)=\gamma^{\prime}(s)$ and call $\boldsymbol{t}(s)$ a unit tangent vector of $\gamma$ at $s$. The curvature of $\gamma$ is defined by $\kappa(s)=\sqrt{\left\|\gamma^{\prime \prime}(s)\right\|}$. If $\kappa(s) \neq 0$, then the unit principal normal vector $\boldsymbol{n}(s)$ of the curve $\gamma$ at $s$ is given by $\gamma^{\prime \prime}(s)=\kappa(s) \boldsymbol{n}(s)$. The unit vector $\boldsymbol{b}(s)=\boldsymbol{t}(s) \times \boldsymbol{n}(s)$ is called a unit binormal vector of the curve $\gamma$ at $s$. Then the following Frenet-Serret formula holds:

$$
\left\{\begin{array}{l}
\boldsymbol{t}^{\prime}(s)=\kappa(s) \boldsymbol{n}(s) \\
\boldsymbol{n}^{\prime}(s)=-\kappa(s) \boldsymbol{t}(s)+\tau(s) \boldsymbol{b}(s) \\
\boldsymbol{b}^{\prime}(s)=-\tau(s) \boldsymbol{n}(s),
\end{array}\right.
$$

where $\tau(s)$ is the torsion of the curve $\gamma$ at $s$. It is well known that the point at where the torsion $\tau(s)$ vanishes is the point at where the curve has at least third order contact with the osculating plane. It corresponds to the degenerated singularities of the tangent developable of $\gamma$ (cf. [3]). So we do not consider such a point for the study of singularities of rectifying developable of $\gamma$. We assume that $\tau(s) \neq 0$ throughout this paper. For any unit speed curve $\gamma: I \longrightarrow \mathbb{R}^{3}$, we call $D(s)=\tau(s) \boldsymbol{t}(s)+\kappa(s) \boldsymbol{b}(s)$ a Darboux vector of $\gamma$ (cf. [7, Section 5.2]). By using the Darboux vector, the Frenet-Serret formula is rewritten 
as follows:

$$
\left\{\begin{array}{l}
\boldsymbol{t}^{\prime}(s)=D(s) \times \boldsymbol{t}(s) \\
\boldsymbol{n}^{\prime}(s)=D(s) \times \boldsymbol{n}(s) \\
\boldsymbol{b}^{\prime}(s)=D(s) \times \boldsymbol{b}(s) .
\end{array}\right.
$$

Thus the Darboux vector plays an important role for the study of space curves. We define a vector $\tilde{D}(s)=(\tau / \kappa)(s) \boldsymbol{t}(s)+\boldsymbol{b}(s)$ and we call it a modified Darboux vector along $\gamma$. We also define a spherical curve $\boldsymbol{d}: I \longrightarrow S^{2}$ by $\boldsymbol{d}(s)=\frac{D(s)}{\|D(s)\|}$ and the surfaces

$$
\begin{gathered}
R G(\gamma)=\{u \boldsymbol{t}(s)+\boldsymbol{b}(s) \mid u \in \mathbb{R}, s \in I\}, \\
R D(\gamma)=\{\gamma(s)+u \tilde{D}(s) \mid u \in \mathbb{R}\} .
\end{gathered}
$$

We call the image of $\boldsymbol{d}$ the spherical Darboux image, the surface $R G(\gamma)$ the rectifying Gaussian surface and the surface $R D(\gamma)$ the rectifying developable of $\gamma$. Our main results are the following:

THEOREM 2.1. Let $\operatorname{Imm}_{r}\left(S^{1}, \mathbb{R}^{3}\right)$ be the space of regular curves $\gamma: S^{1} \longrightarrow \mathbb{R}^{3}$ with $\tau \neq 0$ and $\kappa \neq 0$ equipped with $C^{\infty}$-topology. Then there exists a residual set $\mathcal{O} \subset$ $\operatorname{Imm}_{r}\left(S^{1}, \mathbb{R}^{3}\right)$ such that for any $\gamma \in \mathcal{O}$ the following properties hold:

(1) The number of the points $s \in S^{1}$ where $(\tau / \kappa)^{\prime}(s)=0$ is finite.

(2) There is no point $s \in S^{1}$ where $(\tau / \kappa)^{\prime}(s)=(\tau / \kappa)^{\prime \prime}(s)=0$.

(3) The number of the points $s \in S^{1}$ where $(\tau / \kappa)^{\prime \prime}(s)=0$ is finite.

(4) There is no point $s \in S^{1}$ where $(\tau / \kappa)^{\prime \prime}(s)=(\tau / \kappa)^{\prime \prime \prime}(s)=0$.

THEOREM 2.2. Let $\gamma: I \longrightarrow \mathbb{R}^{3}$ be a unit speed curve. Then we have the following:

(1) The spherical Darboux image is locally diffeomorphic to the ordinary cusp $C$ at $\boldsymbol{d}\left(s_{0}\right)$ if and only if $(\tau / \kappa)^{\prime}\left(s_{0}\right)=0$ and $(\tau / \kappa)^{\prime \prime}\left(s_{0}\right) \neq 0$.

(2) (a) The rectifying Gaussian surface is locally diffeomorphic to the cuspidal edge $C \times \mathbb{R}$ at $u_{0} \boldsymbol{t}\left(s_{0}\right)+\boldsymbol{b}\left(s_{0}\right)$ if and only if $u_{0}=(\tau / \kappa)\left(s_{0}\right)$ and $(\tau / \kappa)^{\prime}\left(s_{0}\right) \neq 0$.

(b) The rectifying Gaussian surface is locally diffeomorphic to the swallow tail $S W$ at $u_{0} \boldsymbol{t}\left(s_{0}\right)+\boldsymbol{b}\left(s_{0}\right)$ if and only if $u_{0}=(\tau / \kappa)\left(s_{0}\right),(\tau / \kappa)^{\prime}\left(s_{0}\right)=0$ and $(\tau / \kappa)^{\prime \prime}\left(s_{0}\right) \neq 0$.

(3) (a) The rectifying developable is locally diffeomorphic to the cuspidal edge $C \times \mathbb{R}$ at $\gamma\left(s_{0}\right)+u_{0} \tilde{D}\left(s_{0}\right)$ if and only if $(\tau / \kappa)^{\prime}\left(s_{0}\right) \neq 0,(\tau / \kappa)^{\prime \prime}\left(s_{0}\right) \neq 0$ and $u_{0}=\frac{1}{(\tau / \kappa)^{\prime}\left(s_{0}\right)}$.

(b) The rectifying developable is locally diffeomorphic to the swallow tail $S W$ at $\gamma\left(s_{0}\right)+u_{0} \tilde{D}\left(s_{0}\right)$ if and only if $(\tau / \kappa)^{\prime}\left(s_{0}\right) \neq 0,(\tau / \kappa)^{\prime \prime}\left(s_{0}\right)=0,(\tau / \kappa)^{\prime \prime \prime}\left(s_{0}\right) \neq 0$ and $u_{0}=\frac{1}{(\tau / \kappa)^{\prime}\left(s_{0}\right)}$.

Here, $C=\left\{\left(x_{1}, x_{2}\right) \mid x_{1}^{2}=x_{2}^{3}\right\}$ is the ordinary cusp and $S W=\left\{\left(x_{1}, x_{2}, x_{3}\right) \mid x_{1}=\right.$ $\left.3 u^{4}+u^{2} v, x_{2}=4 u^{3}+2 u v, x_{3}=v\right\}$ is the swallow tail.

The geometric meanings of the singularities of Image $\boldsymbol{d}, R G(\gamma)$ and $R D(\gamma)$ will be discussed in Section 4.

3. Families of smooth functions on a space curve. In this section we introduce two different families of functions on a space curve which are useful for the study of singularities of $\boldsymbol{d}, R G(\gamma)$ and $R D(\gamma)$. Let $\gamma: I \rightarrow \mathbb{R}^{3}$ be a unit speed space curve with $\kappa(s) \neq 0$ and $\tau(s) \neq 0$. 
Volumelike distance functions. We now define a three-parameter family of smooth functions on $I$ :

$$
F: I \times \mathbb{R}^{3} \rightarrow \mathbb{R}
$$

by $F(s, u)=|\boldsymbol{t}(s) \boldsymbol{n}(s) \gamma(s)-u|=\langle\gamma(s)-u, \boldsymbol{b}(s)\rangle$. Here, $|\boldsymbol{a} \boldsymbol{b} \boldsymbol{c}|$ denotes the determinant of the matrix $(\boldsymbol{a} \boldsymbol{b} \boldsymbol{c})$ and $\langle\boldsymbol{a}, \boldsymbol{b}\rangle$ denotes the standard inner product of $\boldsymbol{a}$ and $\boldsymbol{b}$. We call $F$ a volumelike distance function (or a binormal directed distance function) on $\gamma$. We use the notation $f_{u}(s)=F(s, u)$ for any $u \in \mathbb{R}^{3}$.

Proposition 3.1. Let $\gamma: I \rightarrow \mathbb{R}^{3}$ be a unit speed curve with $\kappa(s) \neq 0$ and $\tau(s) \neq 0$. Then

(1) $f_{u}^{\prime}(s)=0$ if and only if $\gamma(s)-u \in\langle\boldsymbol{t}(s), \boldsymbol{b}(s)\rangle_{\mathbb{R}}$.

(2) $f_{u}^{\prime}(s)=f_{u}^{\prime \prime}(s)=0$ if and only if $\gamma(s)-u=\mu((\tau / \kappa)(s) \boldsymbol{t}(s)+\boldsymbol{b}(s))$.

(3) $f_{u}^{\prime}(s)=f_{u}^{\prime \prime}(s)=f_{u}^{\prime \prime \prime}(s)=0$ if and only if $\gamma(s)-u=\frac{1}{(\tau / \kappa)^{\prime}(s)}((\tau / \kappa)(s) \boldsymbol{t}(s)+\boldsymbol{b}(s))$ and $(\tau / \kappa)^{\prime}(s) \neq 0$.

(4) $f_{u}^{\prime}(s)=f_{u}^{\prime \prime}(s)=f_{u}^{\prime \prime \prime}(s)=f_{u}^{(4)}(s)=0$ if and only if

$$
\gamma(s)-u=\frac{1}{(\tau / \kappa)^{\prime}(s)}((\tau / \kappa)(s) \boldsymbol{t}(s)+\boldsymbol{b}(s)),
$$

$(\tau / \kappa)^{\prime}(s) \neq 0$ and $(\tau / \kappa)^{\prime \prime}(s)=0$.

(5) $f_{u}^{\prime}(s)=f_{u}^{\prime \prime}(s)=f_{u}^{\prime \prime \prime}(s)=f_{u}^{(4)}(s)=f_{u}^{(5)}(s)=0$ if and only if

$$
\gamma(s)-u=\frac{1}{(\tau / \kappa)^{\prime}(s)}((\tau / \kappa)(s) \boldsymbol{t}(s)+\boldsymbol{b}(s)),
$$

$(\tau / \kappa)^{\prime}(s) \neq 0$ and $(\tau / \kappa)^{\prime \prime}(s)=0$ and $(\tau / \kappa)^{\prime \prime \prime}(s)=0$.

P r o of. By the Frenet-Serret formula, we have the following calculation:

(a) $f_{u}^{\prime}(s)=\tau(s)|\boldsymbol{t}(s) \boldsymbol{b}(s) \gamma(s)-u|$.

(b) $f_{u}^{\prime \prime}(s)=-\tau^{2}(s)|\boldsymbol{t}(s) \boldsymbol{n}(s) \gamma(s)-u|+\kappa(s) \tau(s)|\boldsymbol{n}(s) \boldsymbol{b}(s) \gamma(s)-u|$ $+\tau^{\prime}(s)|\boldsymbol{t}(s) \boldsymbol{b}(s) \gamma(s)-u|$.

(c) $f_{u}^{\prime \prime \prime}(s)=-3 \tau(s) \tau^{\prime}(s)|\boldsymbol{t}(s) \boldsymbol{n}(s) \gamma(s)-u|$

$$
\begin{aligned}
& +\left(2 \kappa(s) \tau^{\prime}(s)+\kappa^{\prime}(s) \tau(s)\right)|\boldsymbol{n}(s) \boldsymbol{b}(s) \gamma(s)-u| \\
& +\left(\tau^{\prime \prime}(s)-\kappa^{2}(s) \tau(s)-\tau^{3}(s)\right)|\boldsymbol{t}(s) \boldsymbol{b}(s) \gamma(s)-u|+\kappa(s) \tau(s) .
\end{aligned}
$$

Since $\tau(s) \neq 0$, assertion (1) follows from formula (a) above. By (1) and (b), we have $\gamma\left(s_{0}\right)-u=\nu \boldsymbol{t}\left(s_{0}\right)+\mu \boldsymbol{b}\left(s_{0}\right)$ and

$$
-\tau^{2}(s)|\boldsymbol{t}(s) \boldsymbol{n}(s) \mu \boldsymbol{b}(s)|+\kappa(s) \tau(s)|\boldsymbol{n}(s) \boldsymbol{b}(s) \nu \boldsymbol{t}(s)|=-\tau^{2}(s) \mu+\kappa(s) \tau(s) \nu=0 .
$$

Thus we have $\nu=(\tau / \kappa)(s) \mu$.

By (2) and (c), we have $\gamma(s)-u=\mu((\tau / \kappa)(s) \boldsymbol{t}(s)+\boldsymbol{b}(s))$ and

$$
\begin{aligned}
-\tau(s) \tau^{\prime}(s)|\boldsymbol{t}(s) \boldsymbol{n}(s) \mu \boldsymbol{b}(s)| & +\kappa^{\prime}(s) \tau(s)|\boldsymbol{n}(s) \boldsymbol{b}(s)(\tau / \kappa)(s) \boldsymbol{t}(s)|+\kappa(s) \tau(s) \\
& =\left(-\tau(s) \tau^{\prime}(s)+\left(\kappa^{\prime}(s) \tau^{2}(s) / \kappa(s)\right)\right) \mu+\kappa(s) \tau(s)=0 .
\end{aligned}
$$

This implies that $\frac{\kappa(s) \tau^{\prime}(s)-\kappa^{\prime}(s) \tau(s)}{\kappa(s)} \mu=\kappa(s)$. Hence, we have $(\tau / \kappa)^{\prime}(s) \cdot \mu=1$.

By the similar but rather long calculation of higher derivatives of $f_{u}(s)$, we can also show the remaining assertions. 
We can also define the normally directed distance function on $I$ :

$$
H: I \times \mathbb{R}^{3} \rightarrow \mathbb{R}
$$

by $H(s, u)=\langle\gamma(s)-u, \boldsymbol{n}(s)\rangle$. It is analogous to Example 7.14 (8) of [1]. By definition, $H^{-1}(0)$ gives the one-parameter family of rectifying planes along $\gamma$. So the rectifying developable of $\gamma$ is the discriminant set (cf. Section 5) of $H$. The calculation for the proof of Proposition 3.1 is slightly easier if we adopt $H$ instead of $F$. For the classification of singularities, we must prove the (p) versality (cf. Section 5 ) of $H$. It is, however, much harder than the proof for the versality of $F$ (cf. Section 5, Proposition 5.4). This is the reason why we adopt the volumelike distance function $F$.

Volumelike height functions. We define a two-parameter family of smooth functions on $I$ :

$$
G: I \times S^{2} \rightarrow \mathbb{R}
$$

by $G(s, v)=|\boldsymbol{n}(s) \boldsymbol{b}(s) v|=\langle\boldsymbol{t}(s), v\rangle$. We call $G$ a volumelike height function (or a tangential height function) on $\gamma$ and use the notation $g_{v}(s)=G(s, v)$.

Proposition 3.2. Let $\gamma: I \longrightarrow \mathbb{R}^{3}$ be a unit speed curve with $\kappa(s) \neq 0$ and $\tau(s) \neq 0$. Then

(1) $g_{v}^{\prime}(s)=0$ if and only if there exist real numbers $\lambda, \mu \in \mathbb{R}$, such that $v=\lambda \boldsymbol{t}(s)+$ $\mu \boldsymbol{b}(s), \lambda^{2}+\mu^{2}=1$.

(2) $g_{v}^{\prime}(s)=g_{v}^{\prime \prime}(s)=0$ if and only if $v= \pm \frac{\kappa(s)}{\sqrt{\tau^{2}(s)+\kappa^{2}(s)}}((\tau / \kappa)(s) \boldsymbol{t}(s)+\boldsymbol{b}(s))$.

(3) $g_{v}^{\prime}(s)=g_{v}^{\prime \prime}(s)=g_{v}^{\prime \prime \prime}(s)=0$ if and only if

$$
v= \pm \frac{\kappa(s)}{\sqrt{\tau^{2}(s)+\kappa^{2}(s)}}((\tau / \kappa)(s) \boldsymbol{t}(s)+\boldsymbol{b}(s)),(\tau / \kappa)^{\prime}(s)=0 .
$$

(4) $g_{v}^{\prime}(s)=g_{v}^{\prime \prime}(s)=g_{v}^{\prime \prime \prime}(s)=g_{v}^{(4)}(s)=0$ if and only if

$$
v= \pm \frac{\kappa(s)}{\sqrt{\tau^{2}(s)+\kappa^{2}(s)}}((\tau / \kappa)(s) \boldsymbol{t}(s)+\boldsymbol{b}(s)),(\tau / \kappa)^{\prime}(s)=(\tau / \kappa)^{\prime \prime}(s)=0 .
$$

(5) $g_{v}^{\prime}(s)=g_{v}^{\prime \prime}(s)=g_{v}^{\prime \prime \prime}(s)=g_{v}^{(4)}(s)=g_{v}^{(5)}(s)=0$ if and only if

$$
v= \pm \frac{\kappa(s)}{\sqrt{\tau^{2}(s)+\kappa^{2}(s)}}((\tau / \kappa)(s) \boldsymbol{t}(s)+\boldsymbol{b}(s))
$$

and $(\tau / \kappa)^{\prime}(s)=(\tau / \kappa)^{\prime \prime}(s)=(\tau / \kappa)^{\prime \prime \prime}(s)=0$.

Pro of. By the Frenet-Serret formula, we have the following calculation:

(a) $g_{v}^{\prime}(s)=-\kappa(s)|\boldsymbol{t}(s) \boldsymbol{b}(s) v|$.

(b) $g_{v}^{\prime \prime}(s)=-\kappa^{\prime}(s)|\boldsymbol{t}(s) \boldsymbol{b}(s) v|-\kappa^{2}(s)|\boldsymbol{n}(s) \boldsymbol{b}(s) v|+\kappa(s) \tau(s)|\boldsymbol{t}(s) \boldsymbol{n}(s) v|$.

(c) $g_{v}^{\prime \prime \prime}(s)=\left(-\kappa^{\prime \prime}(s)+\kappa^{3}(s)+\kappa(s) \tau^{2}(s)\right)|\boldsymbol{t}(s) \boldsymbol{b}(s) v|-3 \kappa(s) \kappa^{\prime}(s)|\boldsymbol{n}(s) \boldsymbol{b}(s) v|$

$$
+\left(2 \kappa^{\prime}(s) \tau(s)+\kappa(s) \tau^{\prime}(s)\right)|\boldsymbol{t}(s) \boldsymbol{n}(s) v| .
$$

Assertion (1) is trivial by formula (a) in the above calculation.

(2) By (1), we have $v=\lambda \boldsymbol{t}(s)+\mu \boldsymbol{b}(s)$. It follows from (b) that $g_{v}^{\prime \prime}(s)=\kappa(s)(\kappa(s) \lambda-$ $\tau(s) \mu)$. Since $\kappa(s) \neq 0, g_{v}^{\prime \prime}(s)=0$ if and only if $\lambda=\frac{\tau(s)}{\kappa(s)} \mu$. Therefore we have $v=$ 
$(\tau / \kappa)(s) \mu \boldsymbol{t}(s)+\mu \boldsymbol{b}(s)=\mu((\tau / \kappa)(s) \boldsymbol{t}(s)+\boldsymbol{b}(s))$. By the assumption that $v \in S^{2}$ and $\kappa(s) \geq 0$, we have $\mu= \pm \frac{\kappa(s)}{\sqrt{\tau^{2}(s)+\kappa^{2}(s)}}$.

(3) We now substitute the formula $v= \pm \frac{\kappa(s)}{\sqrt{\tau^{2}(s)+\kappa^{2}(s)}}((\tau / \kappa)(s) \boldsymbol{t}(s)+\boldsymbol{b}(s))$ into (c), then we have

$$
\left(-\kappa^{\prime}(s) \tau(s)+\kappa(s) \tau^{\prime}(s)\right)\left( \pm \frac{\kappa(s)}{\sqrt{\tau^{2}(s)+\kappa^{2}(s)}}\right)=0 .
$$

Since $\pm \frac{\kappa(s)}{\sqrt{\tau^{2}(s)+\kappa^{2}(s)}} \neq 0$ and $(\tau / \kappa)^{\prime}(s)=\left(1 / \kappa^{2}(s)\right)\left(\tau^{\prime}(s) \kappa(s)-\tau(s) \kappa^{\prime}(s)\right)$, assertion (3) follows.

Assertions (4), (5) also follow from the direct calculation.

4. Helices and the spherical tangential image of a curve. In this section we study the geometric properties of the rectifying developable, the spherical Darboux image and the rectifying Gaussian surface of space curves. By the propositions in the last section, we can recognize that the function $(\tau / \kappa)^{\prime}(s)$ and the modified Darboux vector $\tilde{D}(s)=$ $(\tau / \kappa)(s) \boldsymbol{t}(s)+\boldsymbol{b}(s)$ are the important subjects. If $(\tau / \kappa)^{\prime}(s) \equiv 0$ then the curve $\gamma(s)$ has been classically known as a helix. For a unit speed regular curve $\gamma: I \longrightarrow \mathbb{R}^{3}$, the unit tangent curve $\boldsymbol{t}: I \longrightarrow S^{2}$ is called the spherical tangential image of $\gamma$. We can easily calculate that the geodesic curvature of the spherical tangential image $\boldsymbol{t}$ is equal to the function $\tau / \kappa$, which has been called the conical curvature of $\gamma$ (cf. [7, Section 5.2]). Moreover, we have the following proposition.

Proposition 4.1. Let $\gamma: I \longrightarrow \mathbb{R}^{3}$ be a regular curve. Then $\gamma$ is a helix if and only if the modified Darboux vector $\tilde{D}(s)$ is a constant vector. In this case we have the following assertions:

(1) The spherical tangential image $\boldsymbol{t}(s)$ of $\gamma$ is a circle on the unit sphere $S^{2}$ and the direction of the center of the circle is given by the constant vector $\boldsymbol{d}(s) \equiv \boldsymbol{e}$.

(2) The rectifying developable of $\gamma$ is a cylindrical surface given by $\gamma(s)+u \boldsymbol{e}$.

P r o of. By the Frenet-Serret formula, we can show that $\tilde{D}^{\prime}(s)=(\tau / \kappa)^{\prime}(s) \boldsymbol{t}(s)$. Therefore, $\gamma$ is a helix if and only if $\tilde{D}^{\prime}(s) \equiv 0$. This condition is equivalent to the condition that $\tilde{D}(s)$ is a constant vector. In this case we have $\langle\boldsymbol{e}, \boldsymbol{t}(s)\rangle=\cos \theta(s)$, where $\theta(s)$ is the angle between two vectors $\boldsymbol{t}(s), \boldsymbol{e}$. Since

$$
\boldsymbol{e}= \pm \frac{\kappa(s)}{\sqrt{\tau^{2}(s)+\kappa^{2}(s)}}((\tau / \kappa)(s) \boldsymbol{t}(s)+\boldsymbol{b}(s))
$$

we also have $\langle\boldsymbol{e}, \boldsymbol{t}(s)\rangle=\frac{(\tau / \kappa)(s)}{\sqrt{(\tau / \kappa)^{2}(s)+1}}$, so $\cos \theta(s)$ is constant. This means that the spherical tangential image $\boldsymbol{t}(s)$ is a circle on the unit sphere $S^{2}$ and the center is directed by $\boldsymbol{e}$. The assertion (2) is clear by definition.

Let $\gamma_{i}: I_{i} \longrightarrow \mathbb{R}^{3}(i=1,2)$ be regular curves. We say that $\gamma_{1}\left(s_{0}\right)$ and $\gamma_{2}\left(t_{0}\right)$ have at least $(k+1)$-point contact if $\gamma_{1}^{(p)}\left(s_{0}\right)=\gamma_{2}^{(p)}\left(t_{0}\right)$ for $0 \leq p \leq k$. We also say that $\gamma_{1}\left(s_{0}\right)$ and $\gamma_{2}\left(t_{0}\right)$ have $(k+1)$-point contact if they have at least $(k+1)$-point contact and satisfy 
the relation $\gamma_{1}^{(k+1)}\left(s_{0}\right) \neq \gamma_{2}^{(k+1)}\left(t_{0}\right)$. By definition of the curvature and the torsion, we can show the following proposition.

Proposition 4.2. If $\gamma_{1}\left(s_{0}\right)$ and $\gamma_{2}\left(t_{0}\right)$ have $(k+1)$-point contact, then $(\tau / \kappa)_{1}^{(p)}\left(s_{0}\right)=(\tau / \kappa)_{2}^{(p)}\left(t_{0}\right)$ (for $\left.0 \leq p \leq k-3\right)$ and $(\tau / \kappa)_{1}^{(k-2)}\left(s_{0}\right) \neq(\tau / \kappa)_{2}^{(k-2)}\left(t_{0}\right)$.

We also have the following proposition.

Proposition 4.3. Let $\gamma: I \longrightarrow \mathbb{R}^{3}$ be a regular curve with $\kappa\left(s_{0}\right) \neq 0$ and $\tau\left(s_{0}\right) \neq 0$. Then there exists an open interval $s_{0} \in J \subset I$ and a unique helix $\delta: J \longrightarrow \mathbb{R}^{3}$ such that $\delta\left(s_{0}\right)=\gamma\left(s_{0}\right)$, the curvature of $\delta(s)$ is $\kappa(s)$, the torsion of $\delta$ at $s_{0}$ is $\tau\left(s_{0}\right)$ and $\gamma$ and $\delta$ have at least 4-point contact at $s_{0}$.

The proof of Proposition 4.3 is given by solving the natural equation $\kappa_{\delta}(s)=\kappa(s)$, $\tau_{\delta}(s)=(\tau / \kappa)\left(s_{0}\right) \tau(s)$ under the initial condition $\delta\left(s_{0}\right)=\gamma\left(s_{0}\right), \delta^{\prime}\left(s_{0}\right)=\gamma^{\prime}\left(s_{0}\right)$ and $\delta^{\prime \prime \prime}\left(s_{0}\right)=\gamma^{\prime \prime \prime}\left(s_{0}\right)$. So we omit the detail. We call the helix $\delta$ in Proposition 4.3 the osculating helix of $\gamma$ at $s_{0}$. We denote it by $\delta\left[s_{0}\right](s)$. By Proposition 4.1 , the spherical tangential image $\boldsymbol{t}_{\delta\left[s_{0}\right]}(s)$ of the helix $\delta\left[s_{0}\right]$ is a circle whose center is directed by the spherical Darboux image $\boldsymbol{d}\left(s_{0}\right)$ of $\gamma$ at $s_{0}$. We call $\boldsymbol{d}\left(s_{0}\right)$ the center of conical curvature of $\gamma$. We also call the locus of the center of conical curvature of $\gamma$ the spherical (or, conical) evolute of $\gamma$. Under the above terminology, the spherical Darboux image is the spherical evolute of $\gamma$. Therefore the singularities of the spherical Darboux image (or, the rectifying Gaussian surface) describe how the shape of the curve $\gamma$ is similar to a helix. On the other hand, the singularities of the rectifying developable describe how the shape of the curve $\gamma$ is different from a helix.

5. Unfoldings of functions by one-variable. In this section we use some general results on the singularity theory for families of function germs. Detailed descriptions can be found in the book [1]. Let $F:\left(\mathbb{R} \times \mathbb{R}^{r},\left(s_{0}, x_{0}\right)\right) \rightarrow \mathbb{R}$ be a function germ. We call $F$ an $r$-parameter unfolding of $f$, where $f(s)=F_{x_{0}}\left(s, x_{0}\right)$. We say that $f$ has $A_{k}$-singularity at $s_{0}$ if $f^{(p)}\left(s_{0}\right)=0$ for all $1 \leq p \leq k$, and $f^{(k+1)}\left(s_{0}\right) \neq 0$. We also say that $f$ has $A_{\geq k}$-singularity at $s_{0}$ if $f^{(p)}\left(s_{0}\right)=0$ for all $1 \leq p \leq k$. Let $F$ be an unfolding of $f$ and $f(s)$ has $A_{k}$-singularity $(k \geq 1)$ at $s_{0}$. We denote the $(k-1)$-jet of the partial derivative $\frac{\partial F}{\partial x_{i}}$ at $s_{0}$ by $j^{(k-1)}\left(\frac{\partial F}{\partial x_{i}}\left(s, x_{0}\right)\right)\left(s_{0}\right)=\sum_{j=1}^{k-1} \alpha_{j i} s^{j}$ for $i=1, \ldots, r$. Then $F$ is called a $(p)$ versal unfolding if the $(k-1) \times r$ matrix of coefficients $\left(\alpha_{j i}\right)$ has rank $k-1$ $(k-1 \leq r)$. Under the same condition as above, $F$ is called a versal unfolding if the $k \times r$ matrix of coefficients $\left(\alpha_{0 i}, \alpha_{j i}\right)$ has rank $k(k \leq r)$, where $\alpha_{0 i}=\frac{\partial F}{\partial x_{i}}\left(s_{0}, x_{0}\right)$.

We now introduce important sets concerning the unfoldings relative to the above notions. The bifurcation set $\mathcal{B}_{F}$ of $F$ is the set

$$
\mathcal{B}_{F}=\left\{x \in \mathbb{R}^{r} \mid \text { there exists } s \text { with } \frac{\partial F}{\partial s}=\frac{\partial^{2} F}{\partial s^{2}}=0 \text { at }(s, x)\right\}
$$

The discriminant set of $F$ is the set

$$
\mathcal{D}_{F}=\left\{x \in \mathbb{R}^{r} \mid \text { there exists } s \text { with } F=\frac{\partial F}{\partial s}=0 \text { at }(s, x)\right\} .
$$

Then we have the following well known result (cf. [1]). 
THEOREM 5.1. Let $F:\left(\mathbb{R} \times \mathbb{R}^{r},\left(s_{0}, x_{0}\right)\right) \rightarrow \mathbb{R}$ be an r-parameter unfolding of $f(s)$ which has the $A_{k}$ singularity at $s_{0}$.

(1) Suppose that $F$ is a ( $p)$ versal unfolding.

(a) If $k=2$, then $\left(s_{0}, x_{0}\right)$ is the fold point of $\pi \mid S_{F}$ and $\mathcal{B}_{F}$ is locally diffeomorphic to $\{0\} \times \mathbb{R}^{r-1}$.

(b) If $k=3$, then $\mathcal{B}_{F}$ is diffeomorphic to $C \times \mathbb{R}^{r-2}$.

(2) Suppose that $F$ is a versal unfolding.

(a) If $k=1$, then $\mathcal{D}_{F}$ is locally diffeomorphic to $\{0\} \times \mathbb{R}^{r-1}$.

(b) If $k=2$, then $\mathcal{D}_{F}$ is locally diffeomorphic to $C \times \mathbb{R}^{r-2}$.

(c) If $k=3$, then $\mathcal{D}_{F}$ is locally diffeomorphic to $S W \times \mathbb{R}^{r-3}$.

Here, $C=\left\{\left(x_{1}, x_{2}\right) \mid x_{1}^{2}=x_{2}^{3}\right\}$ is the ordinary cusp and $S W=\left\{\left(x_{1}, x_{2}, x_{3}\right) \mid x_{1}=\right.$ $\left.3 u^{4}+u^{2} v, x_{2}=4 u^{3}+2 u v, x_{3}=v\right\}$ is the swallow tail.

For the proof of Theorem 2.2, we have the following key propositions.

Proposition 5.2. Let $G: I \times S^{2} \longrightarrow \mathbb{R}$ be the volumelike height function on a unit speed curve $\gamma(s)$. If $g_{v_{0}}$ has $A_{k}$-singularity $(k=2,3)$ at $s_{0}$, then $G$ is a $(p)$ versal unfolding of $g_{v_{0}}$.

Proof. We define $\gamma(s)=\left(x_{1}(s), x_{2}(s), x_{3}(s)\right)$ and $v=\left(y_{1}, y_{2}, \sqrt{1-y_{1}^{2}-y_{2}^{2}}\right)$. By definition, we have

$$
G(s, v)=\langle\boldsymbol{t}(s), v\rangle=y_{1} x_{1}^{\prime}(s)+y_{2} x_{2}^{\prime}(s)+\sqrt{1-y_{1}^{2}-y_{2}^{2}} x_{3}^{\prime}(s) .
$$

Let $j^{k-1}\left(\partial G / \partial y_{i}\left(s, y_{0}\right)\right)\left(s_{0}\right)$ be the $(k-1)$-jet of $\partial G / \partial y_{i}$ at $s_{0}$; then we have

$$
\begin{aligned}
j^{3}\left(\frac{\partial G}{\partial y_{i}}\left(s, y_{0}\right)\right)\left(s_{0}\right)=( & \left.x_{i}^{\prime \prime}\left(s_{0}\right)-\frac{y_{0, i}}{\sqrt{1-y_{0,1}^{2}-y_{0,2}^{2}}} x_{3}^{\prime \prime}\left(s_{0}\right)\right) s \\
+ & \frac{1}{2}\left(x_{i}^{\prime \prime \prime}\left(s_{0}\right)-\frac{y_{0, i}}{\sqrt{1-y_{0,1}^{2}-y_{0,2}^{2}}} x_{3}^{\prime \prime \prime}\left(s_{0}\right)\right) s^{2} \\
& +\frac{1}{6}\left(x_{i}^{(4)}\left(s_{0}\right)-\frac{y_{0, i}}{\sqrt{1-y_{0,1}^{2}-y_{0,2}^{2}}} x_{3}^{(4)}\left(s_{0}\right)\right) s^{3} .
\end{aligned}
$$

We distinguish two cases.

Case 1 . When $g_{v_{0}}$ has the $A_{2}$-singularity at $s_{0}$, we define the $1 \times 2$-matrix $A$ as follows:

$$
A=\left(x_{1}^{\prime \prime}\left(s_{0}\right)-\frac{y_{0,1}}{\sqrt{1-y_{0,1}^{2}-y_{0,2}^{2}}} x_{3}^{\prime \prime}\left(s_{0}\right) \quad x_{2}^{\prime \prime}\left(s_{0}\right)-\frac{y_{0,2}}{\sqrt{1-y_{0,1}^{2}-y_{0,2}^{2}}} x_{3}^{\prime \prime}\left(s_{0}\right)\right) .
$$

Since $g_{v_{0}}$ has the $A_{2}$-singularity at $s_{0}$, we have

$$
g_{v_{0}}^{\prime}\left(s_{0}\right)=y_{0,1} x_{1}^{\prime \prime}\left(s_{0}\right)+y_{0,2} x_{2}^{\prime \prime}\left(s_{0}\right)+\sqrt{1-y_{0,1}^{2}-y_{0,2}^{2}} x_{3}^{\prime \prime}\left(s_{0}\right)=0 .
$$

By this relation, we have

$$
\|A\|^{2}=x_{1}^{\prime \prime 2}\left(s_{0}\right)+x_{2}^{\prime \prime 2}\left(s_{0}\right)+\left(1+\frac{1}{1-y_{0,1}^{2}-y_{0,2}^{2}}\right) x_{3}^{\prime \prime 2}\left(s_{0}\right) \neq 0 .
$$

Therefore $\operatorname{rank} A=1$. 
Case 2. When $g_{v_{0}}$ has the $A_{3}$-singularity at $s_{0}$, we also require the $2 \times 2$-matrix

$$
B=\left(\begin{array}{ll}
x_{1}^{\prime \prime}\left(s_{0}\right)-\frac{y_{0,1}}{\sqrt{1-y_{0,1}^{2}-y_{0,2}^{2}}} x_{3}^{\prime \prime}\left(s_{0}\right) & x_{2}^{\prime \prime}\left(s_{0}\right)-\frac{y_{0,2}}{\sqrt{1-y_{0,1}^{2}-y_{0,2}^{2}}} x_{3}^{\prime \prime}\left(s_{0}\right) \\
x_{1}^{\prime \prime \prime}\left(s_{0}\right)-\frac{y_{0,1}}{\sqrt{1-y_{0,1}^{2}-y_{0,2}^{2}}} x_{3}^{\prime \prime \prime}\left(s_{0}\right) & x_{2}^{\prime \prime \prime}\left(s_{0}\right)-\frac{y_{0,2}}{\sqrt{1-y_{0,1}^{2}-y_{0,2}^{2}}} x_{3}^{\prime \prime \prime}\left(s_{0}\right)
\end{array}\right)
$$

to be nonsingular. By the direct calculation, we have

$$
\begin{aligned}
\operatorname{det}(B) & =\frac{1}{\sqrt{1-y_{0,1}^{2}-y_{0,2}^{2}}}\left|\begin{array}{ccc}
x_{1}^{\prime \prime}\left(s_{0}\right) & x_{2}^{\prime \prime}\left(s_{0}\right) & x_{3}^{\prime \prime}\left(s_{0}\right) \\
x_{1}^{\prime \prime \prime}\left(s_{0}\right) & x_{2}^{\prime \prime \prime}\left(s_{0}\right) & x_{3}^{\prime \prime \prime}\left(s_{0}\right) \\
y_{0,1} & y_{0,2} & \sqrt{1-y_{0,1}^{2}-y_{0,2}^{2}}
\end{array}\right| \\
& =\frac{1}{\sqrt{1-y_{0,1}^{2}-y_{0,2}^{2}}}\left\langle\gamma^{\prime \prime}\left(s_{0}\right) \times \gamma^{\prime \prime \prime}\left(s_{0}\right), v\right\rangle .
\end{aligned}
$$

Since $\gamma^{\prime \prime}(s)=\kappa(s) \boldsymbol{n}(s)$, we have $\gamma^{\prime \prime \prime}(s)=-\kappa^{2}(s) \boldsymbol{t}(s)+\kappa^{\prime}(s) \boldsymbol{n}(s)+\kappa(s) \tau(s) \boldsymbol{b}(s)$. By the assumption that $g_{v_{0}}$ has the $A_{3}$-singularity at $s_{0}$, we have

$$
v= \pm \frac{\kappa\left(s_{0}\right)}{\sqrt{\tau^{2}\left(s_{0}\right)+\kappa^{2}\left(s_{0}\right)}}\left((\tau / \kappa)\left(s_{0}\right) \boldsymbol{t}\left(s_{0}\right)+\boldsymbol{b}\left(s_{0}\right)\right) .
$$

Substituting these relations to the above equality, we have

$$
\operatorname{det}(B)=\frac{1}{\sqrt{1-y_{0,1}^{2}-y_{0,2}^{2}}}\left( \pm \kappa^{2}\left(s_{0}\right) \sqrt{\tau^{2}\left(s_{0}\right)+\kappa^{2}\left(s_{0}\right)}\right) \neq 0 .
$$

This means that $\operatorname{rank} B=2$.

We define a function $\tilde{G}: I \times S^{2} \times \mathbb{R} \longrightarrow \mathbb{R}$ by

$$
\tilde{G}(s, v, w)=G(s, v)-w .
$$

We also put $g_{v, w}(s)=\tilde{G}(s, v, w)$.

Proposition 5.3. If $g_{v_{0}, w_{0}}$ has $A_{k}$-singularity $(k=1,2,3)$ at $s_{0}$, then $\tilde{G}$ is a versal unfolding of $g_{v_{0}, w_{0}}$.

Proof. Using the same notation as in the above proposition, we have

$$
\tilde{G}\left(s, v, y_{3}\right)=y_{1} x_{1}^{\prime}(s)+y_{2} x_{2}^{\prime}(s)+\left(\sqrt{1-y_{1}^{2}-y_{2}^{2}}\right) x_{3}^{\prime}(s)-y_{3}
$$

Let $j^{k-1}\left(\partial \tilde{G} / \partial y_{i}\right)\left(s, y_{0}\right)\left(s_{0}\right)$ be the $(k-1)$-jet of $\partial \tilde{G} / \partial y_{i}$ at $s_{0}(i=1,2,3)$; then we have

$$
\begin{aligned}
\frac{\partial \tilde{G}}{\partial y_{i}}\left(s_{0}, y_{0}\right)+j^{2}\left(\frac{\partial \tilde{G}}{\partial y_{i}}\left(s, y_{0}\right)\right)\left(s_{0}\right)=x_{i}^{\prime}\left(s_{0}\right) & -\frac{y_{0, i}}{\sqrt{1-y_{0,1}^{2}-y_{0,2}^{2}}} x_{3}^{\prime}\left(s_{0}\right) \\
& +\left(x_{i}^{\prime \prime}\left(s_{0}\right)-\frac{y_{0, i}}{\sqrt{1-y_{0,1}^{2}-y_{0,2}^{2}}} x_{3}^{\prime \prime}\left(s_{0}\right)\right) s \\
& +\frac{1}{2}\left(x_{i}^{\prime \prime \prime}\left(s_{0}\right)-\frac{y_{0, i}}{\sqrt{1-y_{0,1}^{2}-y_{0,2}^{2}}} x_{3}^{\prime \prime \prime}\left(s_{0}\right)\right) s^{2} .
\end{aligned}
$$

We also distinguish three cases. 
Case 1 . When $g_{v_{0}, w_{0}}$ has the $A_{1}$-singularity at $s_{0}$, the rank of $1 \times 3$ matrix

$$
\left(x_{1}^{\prime}\left(s_{0}\right)-\frac{y_{0,1}}{\sqrt{1-y_{0,1}^{2}-y_{0,2}^{2}}} x_{3}^{\prime}\left(s_{0}\right) \quad x_{2}^{\prime}\left(s_{0}\right)-\frac{y_{0,2}}{\sqrt{1-y_{0,1}^{2}-y_{0,2}^{2}}} x_{3}^{\prime}\left(s_{0}\right)-1\right)
$$

is clearly 1.

Case 2 . When $g_{v_{0}, w_{0}}$ has the $A_{2}$-singularity at $s_{0}$, we require the $2 \times 3$-matrix

$$
D=\left(\begin{array}{ccc}
x_{1}^{\prime}\left(s_{0}\right)-\frac{y_{0,1}}{\sqrt{1-y_{0,1}^{2}-y_{0,2}^{2}}} x_{3}^{\prime}\left(s_{0}\right) & x_{2}^{\prime}\left(s_{0}\right)-\frac{y_{0,2}}{\sqrt{1-y_{0,1}^{2}-y_{0,2}^{2}}} x_{3}^{\prime}\left(s_{0}\right) & -1 \\
x_{1}^{\prime \prime}\left(s_{0}\right)-\frac{y_{0,1}}{\sqrt{1-y_{0,1}^{2}-y_{0,2}^{2}}} x_{3}^{\prime \prime}\left(s_{0}\right) & x_{2}^{\prime \prime}\left(s_{0}\right)-\frac{y_{0,2}}{\sqrt{1-y_{0,1}^{2}-y_{0,2}^{2}}} x_{3}^{\prime \prime}\left(s_{0}\right) & 0
\end{array}\right)
$$

to have the maximal rank. By Case 1 in Proposition 5.2, the second line of $D$ does not vanish, so the rank of $D$ is 2 .

Case 3 . When $g_{v_{0}, w_{0}}$ has the $A_{3}$-singularity at $s_{0}$, we consider the $3 \times 3$ matrix

$$
E=\left(\begin{array}{ccc}
x_{1}^{\prime}\left(s_{0}\right)-\frac{y_{0,1}}{\sqrt{1-y_{0,1}^{2}-y_{0,2}^{2}}} x_{3}^{\prime}\left(s_{0}\right) & x_{2}^{\prime}\left(s_{0}\right)-\frac{y_{0,2}}{\sqrt{1-y_{0,1}^{2}-y_{0,2}^{2}}} x_{3}^{\prime}\left(s_{0}\right) & -1 \\
x_{1}^{\prime \prime}\left(s_{0}\right)-\frac{y_{0,1}}{\sqrt{1-y_{0,1}^{2}-y_{0,2}^{2}}} x_{3}^{\prime \prime}\left(s_{0}\right) & x_{2}^{\prime \prime}\left(s_{0}\right)-\frac{y_{0,2}}{\sqrt{1-y_{0,1}^{2}-y_{0,2}^{2}}} x_{3}^{\prime \prime}\left(s_{0}\right) & 0 \\
x_{1}^{\prime \prime \prime}\left(s_{0}\right)-\frac{y_{0,1}}{\sqrt{1-y_{0,1}^{2}-y_{0,2}^{2}}} x_{3}^{\prime \prime \prime}\left(s_{0}\right) & x_{2}^{\prime \prime \prime}\left(s_{0}\right)-\frac{y_{0,2}}{\sqrt{1-y_{0,1}^{2}-y_{0,2}^{2}}} x_{3}^{\prime \prime \prime}\left(s_{0}\right) & 0
\end{array}\right) .
$$

By Case 2 in Proposition 5.2, the rank of $E$ is 3.

For the volumelike distance function $F$, we have the following proposition.

Proposition 5.4. If $f_{u_{0}}$ has $A_{k}$-singularity $(k=2,3,4)$ at $s_{0}$, then $F$ is a $(p)$ versal unfolding of $f_{u_{0}}$.

Although the proof of Proposition 5.4 is given in a similar way as the proof of Proposition 5.2, we need a patience for awfully long calculation. So we omit the proof here.

We can easily show that $\mathcal{B}_{F}=R D(\gamma), \mathcal{B}_{G}=$ Image, $\boldsymbol{d}$ and the connected component of $\mathcal{D}_{\tilde{G}}$ is diffeomorphic to $R G(\gamma)$, so that Theorem 2.2 follows from Propositions 3.1, 3.2, $5.2,5.3,5.4$ and Theorem 5.1.

6. Generic properties of space curves. In this section we consider the notion of Monge-Taylor maps for space curves in Euclidean space (cf. [1]). Let $\gamma: I \longrightarrow \mathbb{R}^{3}$ be a regular curve, with $I$ an open connected subset of the unit circle $S^{1}$, increasing $t$ corresponding the anticlockwise orientation of $S^{1}$. We now choose a smooth family of unit vectors $\boldsymbol{n}(t)$, with $\boldsymbol{n}(t)$ normal to $\gamma$ at $t$, so $\|\boldsymbol{n}(t)\|=1$ and $\langle\boldsymbol{n}(t), \boldsymbol{t}(t)\rangle=0$ for all $t \in I$ (see [1, Chapter 9]). We can obtain a second smooth family of unit vectors $\boldsymbol{b}(t)=\boldsymbol{t}(t) \times \boldsymbol{n}(t)$ normal to $\gamma$ at $t$. We now use the perpendicular lines spanned by $\boldsymbol{t}(t), \boldsymbol{n}(t), \boldsymbol{b}(t)$ as axes at $\gamma(t)$ with the unit points on the axes corresponding to the three given vectors. Note the curve $\gamma(t)$ is not necessarily unit speed, with $\dot{\gamma}\left(t_{0}\right)=0$ at a point $t_{0} \in S^{1}$. We can write $\gamma(I)$ locally as a graph $\left\{\left(\xi, f_{t}(\xi), g_{t}(\xi)\right)\right\}$, with $j^{1} f_{t}(0)=$ $j^{1} g_{t}(0)=0$. If $V_{k}$ denotes the space of polynomials in $\zeta$ of degree $\geq 2$ and $\leq k$ we 
have a map, the Monge-Taylor map for the space curve $\gamma, \mu_{\gamma}: I \longrightarrow V_{k} \times V_{k}$ given by $\mu_{\gamma}(t)=\left(j^{k} f_{t}(0), j^{k} g_{t}(0)\right) .\left(V_{k} \times V_{k}\right.$ can be identified with $\mathbb{R}^{k-1} \times \mathbb{R}^{k-1}=\mathbb{R}^{2(k-1)}$ via the coordinates $\left(a_{2}, \ldots, a_{k}, b_{2}, \ldots, b_{k}\right)$.) Of course $\mu_{\gamma}$ depends rather heavily on our choice of unit normals $\boldsymbol{n}(t)$. Here, $a_{i}(t)=\frac{f_{t}(0)^{(i)}}{i !}, b_{i}(t)=\frac{g_{t}(0)^{(i)}}{i !}(2 \leq i \leq k)$, that is

$$
V_{k} \times V_{k}=\left\{\left(a_{2} \xi^{2}+b_{3} \xi^{3}+\ldots+b_{k} \xi^{k}\right),\left(b_{2} \xi^{2}+b_{3} \xi^{3}+\ldots+a_{k} \xi^{k}\right)\right\} .
$$

Let $P_{k}$ denote the set of maps $\psi: \mathbb{R}^{3} \rightarrow \mathbb{R}^{3}$ of the form

$$
\psi(x, y, z)=\left(\psi_{1}(x, y, z), \psi_{2}(x, y, z), \psi_{3}(x, y, z)\right)
$$

where $\psi_{i}(x, y, z)$ is a polynomial in $x, y$ and $z$ of degree $\leq k$. We may identify $P_{k}$ and the Euclidean space $\mathbb{R}^{(k+1)^{2}}$. This space provides the required deformations of the curve.

To simplify matters we now assume that the curve $\gamma(I)$ is compact, that is $I=S^{1}$. The identity map $1_{\mathbb{R}^{3}}: \mathbb{R}^{3} \rightarrow \mathbb{R}^{3}$ is of course an element of $P_{k}$ (provided $k \geq 1$ ), and by using the compactness of $\gamma\left(S^{1}\right)$ it easily follows that there is a open neighbourhood $U$ of $1_{\mathbb{R}^{3}}$ in $P_{k}$ with the property that if $\psi \in U$ then $\psi \circ \gamma: S^{1} \longrightarrow \mathbb{R}^{3}$ is a regular curve. If we deform the original curve by the map $\psi$, then we can also obtain the required new smooth family of normal vectors $\boldsymbol{n}_{\psi}(t)$ as follows: since the map $\psi: \mathbb{R}^{3} \rightarrow \mathbb{R}^{3}$ is a diffeomorphism on some open set containing $\gamma(I)$, the vector $\boldsymbol{n}(t)$ will be sent to a new vector $D \psi(\gamma(t)) \boldsymbol{n}(t)$ which will be neither zero nor tangent to $\psi \circ \gamma$ at $t$. Orthogonally projecting this vector onto the normal plane to $\psi \circ \gamma$ at $t$ and normalizing, that is

$$
\boldsymbol{n}_{\psi}(t)=\frac{D \psi(\gamma(t)) \boldsymbol{n}(t)-\left\langle D \psi(\gamma(t)) \boldsymbol{n}(t), \boldsymbol{t}_{\psi}\right\rangle \boldsymbol{t}_{\psi}}{\left\|D \psi(\gamma(t)) \boldsymbol{n}(t)-\left\langle D \psi(\gamma(t)) \boldsymbol{n}(t), \boldsymbol{t}_{\psi}\right\rangle \boldsymbol{t}_{\psi}\right\|}, \quad\left\langle\boldsymbol{n}_{\psi}(t), \boldsymbol{n}_{\psi}(t)\right\rangle=-1,
$$

where $\boldsymbol{t}_{\psi}$ denotes the tangent vector of the curve $\psi \circ \gamma$ at $t$. We choose an open neighbourhood $U$ of $1_{S^{1}} \in P_{k}$ consisting of polynomial maps which map an open set containing $\gamma\left(S^{1}\right)$ diffeomorphically to its image. We have now shown that there is a smooth map

$$
\mu: S^{1} \times U \longrightarrow V_{k} \times V_{k}
$$

defined by $\mu(-, \psi)=$ Monge-Taylor map for the curve $\psi \circ \gamma$ using the family of normal vectors $\boldsymbol{n}_{\psi}(t)$. Then we have the following theorem (cf. Theorem 9.9 in [1]).

TheOREM 6.1. Let $Q$ be a submanifold in $V_{k} \times V_{k}=\mathbb{R}^{2 k-2}$. For some open set $U_{1} \subset U$ containing the identity map, the map $\mu: S^{1} \times U_{1} \rightarrow V_{k} \times V_{k}$ defined by $\mu(t, \psi)=\mu_{\psi \circ \gamma}(t)$ is transverse to $Q$.

By the direct calculations, we have the following lemmas. The calculations are rather long and tedious so we omit details.

LEMMA 6.2. Let $\gamma: S^{1} \rightarrow \mathbb{R}^{3}$ be a curve defined by

$$
\gamma(t)=\left(\xi, f_{t}(\xi), g_{t}(\xi)\right)=\left(\xi, a_{2} \xi^{2}+a_{3} \xi^{3}+\ldots, b_{2} \xi^{2}+b_{3} \xi^{3}+\ldots\right)
$$

with $\xi\left(t_{0}\right)=0$. Then

(1) $k=0$ at $t_{0}$ if and only if $a_{2}{ }^{2}-b_{2}{ }^{2}=0$.

(2) $(\kappa / \tau)^{\prime}\left(t_{0}\right)=0$ if and only if $f_{1}\left(a_{2}, a_{3}, a_{4}, b_{2}, b_{3}, b_{4}\right)=0$, where

$$
f_{1}=4\left(a_{2}^{2}+b_{2}^{2}\right)\left(a_{2} b_{4}-a_{4} b_{2}\right)-9\left(a_{2} a_{3}+b_{2} b_{3}\right)\left(a_{2} b_{3}-a_{3} b_{2}\right) .
$$


(3) $(\kappa / \tau)^{\prime \prime}\left(t_{0}\right)=0$ if and only if $f_{2}\left(a_{2}, a_{3}, a_{4}, a_{5}, b_{2}, b_{3}, b_{4}, b_{5}\right)=0$, where

$$
\begin{aligned}
f_{2}= & 12\left(a_{2}^{2}+b_{2}^{2}\right)^{3}\left(a_{2} b_{3}-a_{3} b_{2}\right)-36\left(a_{2}^{2}+b_{2}^{2}\right)\left(a_{2} a_{4}+b_{2} b_{4}\right)\left(a_{2} b_{3}-a_{3} b_{2}\right) \\
& -27\left(a_{3}^{2}+b_{3}^{2}\right)\left(a_{2}^{2}+b_{2}^{2}\right)\left(a_{2} b_{3}-a_{3} b_{2}\right)+135\left(a_{2} a_{3}+b_{2} b_{3}\right)^{2}\left(a_{2} b_{3}-a_{3} b_{2}\right) \\
& -72\left(a_{2} a_{3}+b_{2} b_{3}\right)\left(a_{2} b_{4}-a_{4} b_{2}\right)\left(a_{2}^{2}+b_{2}^{2}\right)+12\left(a_{3} b_{4}-a_{4} b_{3}\right)\left(a_{2}^{2}+b_{2}^{2}\right)^{2} \\
& +20\left(a_{2} b_{5}-a_{5} b_{2}\right)\left(a_{2}^{2}+b_{2}^{2}\right)^{2} .
\end{aligned}
$$

(4) $(\kappa / \tau)^{\prime \prime \prime}\left(t_{0}\right)=0$ if and only if $f_{3}\left(a_{2}, a_{3}, a_{4}, a_{5}, a_{6}, b_{2}, b_{3}, b_{4}, b_{5}, a_{6}\right)=0$, where

$$
\begin{aligned}
f_{3}= & -18 \cdot 4\left(a_{2} a_{3}+b_{2} b_{3}\right)\left(a_{2} b_{3}-a_{3} b_{2}\right)\left(a_{2}^{2}+b_{2}^{2}\right)^{3} \\
& -60\left(a_{2} a_{5}+b_{2} b_{5}\right)\left(a_{2} b_{3}-a_{3} b_{2}\right)\left(a_{2}^{2}+b_{2}^{2}\right)^{2} \\
& -3^{2} \cdot 12\left(a_{3} a_{4}+b_{3} b_{4}\right)\left(a_{2} b_{3}-a_{3} b_{2}\right)\left(a_{2}^{2}+b_{2}^{2}\right)^{2} \\
& +3 \cdot 15 \cdot 12\left(a_{2} a_{3}+b_{2} b_{3}\right)\left(a_{2} a_{4}+b_{2} b_{4}\right)\left(a_{2} b_{3}-a_{3} b_{2}\right)\left(a_{2}^{2}+b_{2}^{2}\right) \\
& +3 \cdot 15 \cdot 9\left(a_{2} a_{3}+b_{2} b_{3}\right)\left(a_{3}^{2}+b_{3}^{2}\right)\left(a_{2} b_{3}-a_{3} b_{2}\right)\left(a_{2}^{2}+b_{2}^{2}\right) \\
& -3^{3} \cdot 35\left(a_{2} a_{3}+b_{2} b_{3}\right)^{3}\left(a_{2} b_{3}-a_{3} b_{2}\right)+3 \cdot 4^{2}\left(a_{2}^{2}+b_{2}^{2}\right)^{4}\left(a_{2} b_{4}-a_{4} b_{2}\right) \\
& -9 \cdot 4^{2}\left(a_{2} a_{4}+b_{2} b_{4}\right)\left(a_{2} b_{4}-a_{4} b_{2}\right)\left(a_{2}^{2}+b_{2}^{2}\right)^{2} \\
& -9 \cdot 3 \cdot 4\left(a_{3}^{2}+b_{3}^{2}\right)\left(a_{2} b_{4}-a_{4} b_{2}\right)\left(a_{2}^{2}+b_{2}^{2}\right)^{2} \\
& +45 \cdot 12\left(a_{2} a_{3}+b_{2} b_{3}\right)^{2}\left(a_{2} b_{4}-a_{4} b_{2}\right)\left(a_{2}^{2}+b_{2}^{2}\right) \\
& -9 \cdot 12\left(a_{2} a_{3}+b_{2} b_{3}\right)\left(a_{3} b_{4}-a_{4} b_{3}\right)\left(a_{2}^{2}+b_{2}^{2}\right)^{2} \\
& -9 \cdot 20\left(a_{2} a_{3}+b_{2} b_{3}\right)\left(a_{2} b_{5}-a_{5} b_{2}\right)\left(a_{2}^{2}+b_{2}^{2}\right)^{2}+2 \cdot 5 \cdot 4\left(a_{3} b_{5}-a_{5} b_{3}\right)\left(a_{2}^{2}+b_{2}^{2}\right)^{3} \\
& +10 \cdot 4\left(a_{2} b_{6}-a_{6} b_{2}\right)\left(a_{2}^{2}+b_{2}^{2}\right)^{3} .
\end{aligned}
$$

Here $\xi$ is the coordinate along the $\boldsymbol{t}$-direction, $f_{t}(\xi)$ along the $\boldsymbol{n}$-direction and $g_{t}(\xi)$ along the $\boldsymbol{b}$-direction.

LEMMA 6.3. Under the same notation as in Lemma 6.2, we have the following assertions:

(1) The rank of the Jacobi matrix $\frac{\partial\left(f_{1}, f_{2}\right)}{\partial\left(a_{i}, b_{i} ; i=1, \ldots, 5\right)}$ is two.

(2) The rank of the Jacobi matrix $\frac{\partial\left(f_{2}, f_{3}\right)}{\partial\left(a_{i}, a_{i} ; i=1, \ldots, 6\right)}$ is two.

The proof of Theorem 2.1 follows from Theorem 6.1 and Lemmas 6.2, 6.3.

\section{References}

[1] J. W. Bruce, P. J. Giblin, Curves and Singularities, 2nd ed., Cambridge Univ. Press, Cambridge, 1992.

[2] M. P. do Carmo, Differential Geometry of Curves and Surfaces, Prentice-Hall, Englewood Cliffs, 1976.

[3] J. P. Cleave, The form of the tangent developable at points of zero torsion on space curves, Math. Proc. Cambridge Philos. Soc. 88 (1980), 403-407.

[4] G. Ishikawa, Determinacy of envelope of the osculating hyperplanes to a curve, Bull. London Math. Soc. 25 (1993), 603-610. 
[5] G. Ishikawa, Developable of a curve and its determinacy relative to the osculation-type, Quart. J. Math. Oxford Ser. (2) 46 (1995), 437-451.

[6] G. Is hikawa, Topological classification of the tangent developable of space curves, Hokkaido Univ. Preprint Series 341 (1996).

[7] J. Koenderink, Solid Shape, MIT Press, Cambridge, MA, 1990.

[8] D. Mond, On the tangent developable of a space curve, Math. Proc. Cambridge Philos. Soc. 91 (1982), 351-355.

[9] D. Mond, Singularities of the tangent developable surface of a space curve, Quart. J. Math. Oxford Ser. (2) 40 (1989), 79-91.

[10] I. R. Porteous, The normal singularities of submanifold, J. Differential Geom. 5 (1971), 543-564.

[11] I. R. Porteous, Geometric Differentiation for the Intelligence of Curves and Surfaces, Cambridge Univ. Press, Cambridge, 1994.

[12] O. P. Shcherbak, Projectively dual space curves and Legendre singularities (in Russian), Trudy Tbiliss. Univ. 232-233 (1982), 280-336; English translation: Selecta Math. Soviet. 5 (1986), 391-421. 